\title{
Life-time prevalence and psychosocial correlates of adolescent direct self-injurious behavior: A comparative study of findings in 11 European countries
}

\begin{abstract}
Romuald Brunner, ${ }^{, 1}$ Michael Kaess, ${ }^{, 1,2}$ Peter Parzer, ${ }^{1}$ Gloria Fischer, ${ }^{1}$ Vladimir Carli, ${ }^{3}$ Christina W. Hoven, ${ }^{4,5}$ Camilla Wasserman, ${ }^{4,6}$ Marco Sarchiapone, ${ }^{6}$ Franz Resch, ${ }^{1}$ Alan Apter, ${ }^{7}$ Judith Balazs, ${ }^{8,9}$ Shira Barzilay, ${ }^{7}$ Julio Bobes, ${ }^{10}$ Paul Corcoran, ${ }^{11}$ Doina Cosmanm, ${ }^{12}$ Christian Haring, ${ }^{13}$ Miriam Iosuec, ${ }^{6}$ Jean-Pierre Kahn, ${ }^{14}$ Helen Keeley, ${ }^{11}$ Gergely Meszaros, ${ }^{8}$ Bogdan Nemes, ${ }^{12}$ Tina Podlogar, ${ }^{15}$ Vita Postuvan, ${ }^{15}$ Pilar A. Saiz, ${ }^{10}$ Merike Sisask, ${ }^{16}$ Alexandra Tubiana, ${ }^{14}$ Airi Varnik, ${ }^{16}$ and Danuta Wasserman ${ }^{3}$

${ }^{1}$ Clinic of Child and Adolescent Psychiatry, Centre of Psychosocial Medicine, University of Heidelberg, Heidelberg, Germany; ${ }^{2}$ Orygen Youth Health, Melbourne, VIC, Australia; ${ }^{3}$ National Centre for Suicide Research and Prevention of Mental Ill-Health (NASP), Karolinska Institute, Stockholm, Sweden; ${ }^{4}$ Department of Child and Adolescent Psychiatry, New York State Psychiatric Institute, Columbia University, New York, NY, USA; ${ }^{5}$ Department of Epidemiology, Mailman School of Public Health, Columbia University, New York, NY, USA; ${ }^{6}$ Department of Health Sciences, University of Molise, Campobasso, Italy; ${ }^{7}$ Feinberg Child Study Centre, Schneider Children's Medical Centre, Tel Aviv University, Tel Aviv, Israel; ${ }^{8}$ Vadaskert Child and Adolescent Psychiatric Hospital, Budapest, Hungary; ${ }^{9}$ Institute of Psychology, Eotvos Lorand University, Budapest, Hungary; ${ }^{10}$ Department of Psychiatry, School of Medicine, University of Oviedo; Centro de Investigación Biomédica en Red de Salud Mental, CIBERSAM, Oviedo, Spain; ${ }^{11}$ National Suicide Research Foundation, Cork, Ireland; ${ }^{12}$ Clinical Psychology Department, Iuliu Hatieganu University of Medicine and Pharmacy, Cluj-Napoca, Romania; ${ }^{13}$ Research Division for Mental Health, University for Medical Information Technology (UMIT), Hall in Tirol, Austria; ${ }^{14}$ Department of Psychiatry and Clinical Psychology, Centre Hospitalo-Universitaire (CHU) de NANCY, Universite de Lorraine, Nancy, France; ${ }^{15}$ Slovene Center for Suicide Research, UPIAM, University of Primorska, Koper, Slovenia; ${ }^{16}$ Estonian-Swedish Mental Health \& Suicidology Institute, Estonian Centre of Excellence of Behavioural and Health Sciences, Tallinn University, Tallinn, Estonia
\end{abstract}

\begin{abstract}
Objectives: To investigate the prevalence and associated psychosocial factors of occasional and repetitive direct self-injurious behavior (D-SIB), such as self-cutting, -burning, -biting, -hitting, and skin damage by other methods, in representative adolescent samples from 11 European countries. Methods: Cross-sectional assessment of adolescents was performed within the European Union funded project, Saving and Empowering Young Lives in Europe (SEYLE), which was conducted in 11 European countries. The representative sample comprised 12,068 adolescents (F/M: 6,717/5,351; mean age: $14.9 \pm 0.89$ ) recruited from randomly selected schools. Frequency of D-SIB was assessed by a modified 6-item questionnaire based on previously used versions of the Deliberate Self-Harm Inventory (DSHI). In addition, a broad range of demographic, social, and psychological factors was assessed. Results: Overall lifetime prevalence of D-SIB was 27.6\%; $19.7 \%$ reported occasional D-SIB and $7.8 \%$ repetitive D-SIB. Lifetime prevalence ranged from $17.1 \%$ to $38.6 \%$ across countries. Estonia, France, Germany, and Israel had the highest lifetime rates of D-SIB, while students from Hungary, Ireland, and Italy reported low rates. Suicidality as well as anxiety and depressive symptoms had the highest odds ratios for both occasional and repetitive D-SIB. There was a strong association of D-SIB with both psychopathology and risk-behaviors, including family related neglect and peer-related rejection/victimization. Associations between psychosocial variables and D-SIB were strongly influenced by both gender and country. Only a minor proportion of the adolescents who reported D-SIB ever received medical treatment. Conclusion: These results suggest high lifetime prevalence of D-SIB in European adolescents. Prevalence as well as psychosocial correlates seems to be significantly influenced by both gender and country. These results support the need for a multidimensional approach to better understand the development of SIB and facilitate culturally adapted prevention/intervention. Keywords: Direct self-injurious behavior, self-harm, nonsuicidal self-injury, psychopathology, gender, adolescents, suicide.
\end{abstract}

\section{Introduction}

At the broadest level, all behaviors that are performed intentionally, and with the knowledge that they can or will result in some degree of physical or psychological injury to oneself, could be conceptualized as self-inju-

\footnotetext{
*Both authors contributed equally to this paper therefore both should be considered as first authors.
}

Conflict of interest statement: No conflicts declared. rious or self-harm behavior (Nock, 2010). During the past decades, various terms have been used to describe and define such human self-injury. Nonsuicidal self-injury (NSSI), which is now clearly defined in the section 3 of the new Diagnostic and Statistical Manual of Mental Disorders (DSM-5), is described as intentional self-inflicted damage to the surface of an individual's body without conscious suicidal intent (American Psychiatric Association, 2013). Frequently the term 'deliberate self-harm' (DSH) has been used 
synonymously with both self-injury and even NSSI. However, there are important differences in the definition of DSH and NSSI: (a) DSH includes suicidal behavior (Hawton \& James, 2005; Muehlenkamp, Claes, Havertape, \& Plener, 2012); (b) the definition of DSH commonly includes indirect damage to an individual's body as well (e.g., severe substance abuse, taking overdoses, or ingestion of sharp implements).

There has been an on-going discussion whether determination of a person's intent during self-injury can reliably be performed, especially within the adolescent population and using self-report measures. Kapur, Cooper, O'Connor and Hawton (2013) have recently questioned the concept of NSSI due to its strong association with suicidal behavior. We propose that self-injuring adolescents may often do this with ambivalent suicidal intent and that intent should not be considered as a categorical construct, but rather represented as a continuum. However, it is possible to differentiate between direct self-injury to an individual's body surface, which typically involves cutting or carving the skin (Nock, 2010), but also other forms like self-biting, hitting self on purpose, or burning skin (Lloyd-Richardson, Perrine, Dierker, \& Kelley, 2007), and indirect harm to an individual as defined within the term of DSH. Therefore, this study will focus on direct self-injurious behavior (D-SIB), which is defined as intentional self-inflicted damage to the surface of an individual's body, which includes self-cutting, -burning, -biting, -hitting, and skin damage by other methods, regardless of the suicidal intent.

In community samples, approximately $13 \%-45 \%$ of adolescents reported to engage in self-injury during their lifetime (Nock, 2010). It remains unclear to what extent these variations represent true intercultural variation in the prevalence of self-injury, rather than an artifact caused by differences in the definition of self-injury, assessment tools, study samples and methods. Nock (2010) argued that the wide variation in prevalence estimates of self-injury is largely caused by the fact that measures of self-injury have not been included in any of the large-scale epidemiologic surveys that have generated mental and physical disorder prevalence estimates. Consequently, estimates have been based on small, regional studies that vary according to the above noted confounding factors. According to two international studies on the intercultural prevalence of NSSI, comparable prevalence rates among adolescents from different countries have been suggested (Giletta, Scholte, Engels, Ciairano, \& Prinstein, 2012; Plener, Libal, Keller, Fegert, \& Muehlenkamp, 2009). However, two other studies on this topic of DSH revealed intercultural differences with regards to both self-injurious thoughts (Kokkevi, Rotsika, Arapaki \& Richardson, 2012) and behavior (Madge et al., 2008). Thus, data on this topic remains conflicting, and a recent review concluded that research would benefit from adopting a common approach to assessment to aide cross-cultural studies and comparisons (Muehlenkamp et al., 2012).

Although the prevalence of self-injury seems generally high, there is a substantial amount of adolescents who only engage in low frequent self-harm during a short episode in their lifetime, and another group that rather engages in repetitive self-harm, which has been shown to be associated with a higher load of psychological problems (Brunner et al., 2007). In general, self-injury has been shown to be associated with a broad variety of socio-demographic and psychological factors (Nock, 2010). Female gender has been shown to be associated with higher prevalence of self-injury (Brunner et al., 2007; O'Connor, Rasmussen, Miles, \& Hawton, 2009; Plener et al., 2009). Because self-injury typically first occurs between ages 10-20 years (Whitlock, Eckenrode ,\& Silverman, 2006), higher mean age would be hypothesized to increase lifetime prevalence. Socioeconomic status has been reported to influence the prevalence of self-harm (Brunner et al., 2007; Hilt, Cha, \& Nolen-Hoeksema, 2008; Nada-Raja, Skegg, Langley, Morrison, \& Sowerby, 2004), but remains controversial since high prevalence occurs in various samples consisting mainly of Caucasian adolescents from middle to upper class (Yates, Tracy, \& Luthar, 2008). Regarding the role of parenting and family in the development of self-injury, data are much less controversial. Experiences of sexual, physical, or emotional abuse (Muehlenkamp, Kerr, Bradley, \& Adams Larsen, 2010; Weierich \& Nock, 2008; Yates, Carlson, \& Egeland, 2008), but also family discord and parental criticism have been found to be closely linked to NSSI (Kaess et al., 2012, 2013; Wedig \& Nock, 2007; Yates, Tracy et al., 2008). Peer relationship problems also seem to be associated with NSSI (Giletta et al., 2012). Self-injury is well-known to be positively correlated with a variety of adolescent risk behaviors (e.g., smoking, binge drinking, truancy), as well as comorbid mental health problems (e.g., depression, anxiety, conduct disorder, suicidal ideation) (Brunner et al., 2007; Gollust, Eisenberg, \& Golberstein, 2008; Haw, Hawton, Casey, Bale, \& Shepherd, 2005; Serras, Saules, Cranford, \& Eisenberg, 2010). In general, all of these factors could potentially influence or bias the prevalence estimate of self-injury, where there is an uneven distribution between study samples. In fact, a recent study provided first evidence of a country-specific impact of psychosocial variables (e.g., substance abuse) on self-harm thoughts and suicide attempts (Kokkevi et al., 2012). In addition, it is important to distinguish the influence of these factors while differentiating occasional from repetitive self-injury.

Nock (2010) argued that obtaining accurate estimates of the rate of self-injury in community and clinical samples is essential for understanding the scope of this problem, allocating services and other resources, and for monitoring changes in this behavior overtime. Therefore, the aim of this study was to 
(a) map the lifetime prevalence (differentiated into occasional and repetitive forms) and methods of D-SIB among adolescents in different European countries by utilizing a homogenous methodology; (b) to examine potential cross-national differences and their association with gender, age, household composition, parental and peer relationships, comorbid risk-behaviors and mental health problems; and (c) to investigate interactions of countries and psychosocial correlates to detect particular cultural-specific influences of psychosocial factors on D-SIB.

\section{Methods \\ Description of study sample}

The study was conducted within the framework of the EU funded project, Saving and Empowering Young Lives in Europe (SEYLE). The detailed protocol of the SEYLE study [registered at the US National Institute of Health $(\mathrm{NIH})$ clinical trial registry (NCT00906620), and the German Clinical Trials Register (DRKS00000214)] has been published elsewhere (Wasserman et al., 2010). The main study comprises a sample of 12,395 adolescents recruited from 179 randomly selected schools, within 11 study sites, in the following European countries: Austria, Estonia, France, Germany, Hungary, Ireland, Israel, Italy, Romania, Slovenia, and Spain, with Sweden serving as the coordinating center. In each country, a list of all eligible schools, within the study sites, was generated according to specific inclusion and exclusion criteria (Wasserman et al., 2010).

The response rate of the elected schools was $67.8 \%$; there was no significant difference in school size (except in Slovenia) between nonparticipating and participating schools (Carli et al., 2013). Of the 14,115 students who consented to participate, 1,720 were absent the day of the survey. This resulted in a total of 12,395 students who completed the questionnaire. An additional 327 subjects were excluded based on missing D-SIB data in the questionnaire. This resulted in a total sample of 12,068 adolescents (F/M: 6,717/5,351; mean age: $14.9 \pm$ 0.89 ) included in these analyses. The overall consent rate within the study was $49 \%(14,117$ pupils with consent out of 28,889$)$. In eight countries, the consent rate of the pupils and their parents/caregivers was $76 \%(10,665$ pupils with consent of 14,086 approached). In the three remaining countries, the consent rate was only $23 \%(3,452$ pupils with consent of 14,803 approached) due to national requirements of their local ethics committees (i.e. both parents had to sign the consent form) (Carli et al., 2013).

An analysis with regard to the representativeness of the SEYLE study sites revealed that the demographic characteristics (such as income, immigrant rate, unemployment rate) of the participating study sites were reasonably representative of their respective national population (Carli et al., 2013).

\section{Study procedures}

Ethical approval was obtained from the local ethical committees at each site before commencing the study. Subjects were included into the study only if both pupils and caregivers had given their written consent. Caregivers and children were informed about the purposes, content as well as risks and benefits of the study by a letter, and an accompanying separate consent form, which was collected by the survey personnel before the study's baseline assessment.

All questionnaires were administered in the official language(s) of the respective country and culturally adapted, if required. Cultural adaption was only required if professional translations resulted in cultural inappropriateness and compromised feasibility and acceptability of the assessment. This adaption process involved experienced researchers and clinicians who were native speakers of the respective language, and every cultural adaption was documented and sent to the translation coordinator of the SEYLE project for approval. This procedure aimed to make sure that cultural adaptation never changed the core structure and content of each assessment instrument or intervention program.

The self-report assessment took up to $90 \mathrm{~min}$. Students were supervised by research staff during the assessment, and could ask questions with regards to a better understanding of the questions. The self-report data analyzed for the SEYLE study was collected between October 2009 and December 2010.

\section{Measurements}

The 6-item questionnaire used in the SEYLE study to assess pupils who engaged in direct self-injurious behavior (D-SIB) refers to the intentional self-inflicted damage of the surface of an individual's body by self-cutting, -burning, -hitting, -biting, and skin damage by other methods. This 6-item questionnaire is based on the 9-item DSHI questionnaire from Bjärehed and Lundh (2008), which is a shortened version of the 16-item DSHI by Lundh, Karim, and Quilisch (2007) that originated from the original 17-item DSHI by Gratz (2001). The modified version comprised the same facets on frequency, severity and duration; however, self-injurious acts were combined to simplify and shorten the measure and assess direct self-injury to one's body surface only.

The following 6-items were used in SEYLE: (a) Have you ever intentionally cut your wrist, arms, or other area(s) of your body, or stuck sharp objects into your skin such as needles, pins, staples (NOT INCLUDING tattoos, ear piercing, needles used for drugs, or body piercing)? (b) Have you ever intentionally burned yourself with a cigarette, lighter, or match? (c) Have you ever intentionally carved words, pictures, designs, or other markings into your skin, 
or scratched yourself to the extent that scarring or bleeding occurred? (d) Have you ever intentionally prevented wounds from healing, or bit yourself to the extent that it broke skin? (e) Have you ever intentionally banged your head or punched yourself thereby causing a bruise? (f) Have you ever intentionally hurt yourself in any of the above-mentioned ways so that it led to hospitalization or injury severe enough to require medical treatment? Occasional D-SIB was defined as 1-4 reported lifetime acts of D-SIB; repetitive D-SIB was defined as $\geq 5$ previous events of D-SIB acts during lifetime. The cut-off of $\geq 5$ has been chosen according to the diagnostic criteria of frequency in the new proposed diagnostic entity of NSSI according to DSM-5 (American Psychiatric Association, 2013).

Moreover, data were collected on demographics, household composition, place of birth, parental involvement, peer relationships, parental unemployment, and religiosity using questions procured from the Global School-Based Pupil Health Survey (GSHS) (WHO, 2009) and European Values Study (EVS) (EVS, 2009). Data on different comorbid risk behaviors and psychopathology were assessed using questions from the Global School-Based Student Health Survey (GSHS) (WHO, 2009), the Beck Depression Inventory (BDI-II) (Beck, Steer, Ball, \& Ranieri, 1996), the Zung Self-Rating Anxiety Scale (SAS) (Zung, 1971), and the Paykel Suicide Scale (PSS) (Paykel, Myers, Lindenthal, \& Tanner, 1974).

All psychosocial variables were dichotomized according to cut-off criteria previously defined and published for the SEYLE study (Wasserman et al., 2010), and can also be found in the Table S1. Defined cut-offs for all variables had been established to sensitively detect at-risk students.

\section{Statistical analyses}

All analyses were calculated on lifetime prevalence of D-SIB. However, additional categories of occasional and repetitive self-harm was used for description of prevalence as well as regression of psychosocial variables on D-SIB only. These categories were not used for further calculations for reasons due to increased complexity and difficulties of interpretability.

Descriptive analysis was used to determine the prevalence of D-SIB, and was calculated separately for each gender and country, as well as for gender according to method. Lifetime prevalence was compared between countries by Bonferroni adjusted Wald tests after a multinomial logistic regression analysis with D-SIB categories as the dependent variable, with country as the explanatory factor. Univariate and multivariate multinomial logistic regression analyses were calculated with D-SIB (lifetime, occasional and repetitive) as the dependent variable and psychosocial factors as explanatory variables. Relative Risk Ratios (RRR) and 95\% confidence intervals $(95 \% \mathrm{CIs})$ are presented. In addition, psychosocial factor $\times$ gender and psychosocial factor $\times$ country interactions were calculated within a multiple logistic regression with factor, gender and country as explanatory variables. In $16.7 \%$ of the subjects, at least one missing value in the explanatory variables occurred in the regression analysis. To prevent estimation bias resulting from the exclusion of these subjects, missing values were replaced with imputed values, using the multivariate imputation by chained equations algorithm (van Buuren \& Oudshoorn, 1999). The regression was then calculated for the 20 imputed datasets and the results were combined (Rubin, 2004). Finally, the frequencies of medical treatment due to D-SIB were calculated separately for each country by D-SIB. Because even small effects are statistically significant in large samples the presentation of the results focuses on the report of effect sizes.

\section{Results \\ Prevalence of $D-S I B$}

Prevalence of D-SIB for each gender and country separately is presented in Table 1 . The lifetime prevalence of D-SIB was $27.56 \%(n=3,326)$. Prevalence of occasional D-SIB was $19.73 \%(n=2,381)$, prevalence of repetitive D-SIB was $7.83 \%(n=945)$. In total, significantly more females were reporting D-SIB $\left[\chi^{2}(2)=41.99 ; p<.001\right]$. In most countries, female gender was significantly associated with higher rates of both occasional and repetitive D-SIB. With regards to occasional D-SIB, no gender differences were found in Hungary, Ireland, Israel, Italy, and Romania. With regards to repetitive D-SIB, no gender differences were found in Austria, Ireland, Israel, and Romania. In Italy, males even showed significant higher rates of repetitive D-SIB $(p=.015)$.

Prevalence of D-SIB ranged from $17.12 \%$ to $38.55 \%$ with countries differing significantly in rates of D-SIB $\left[\chi^{2}(10)=266.96 ; p<.001\right]$. France, Germany, Estonia, and Israel showed the highest lifetime rates of D-SIB, while students from Hungary, Ireland, Romania, and Italy reported low lifetime rates of D-SIB; $p$-values indicated significant differences for total D-SIB between countries and are presented in Table S2 of this article. Prevalence of occasional D-SIB ranged from $12.51 \%$ to $25.60 \%$ with countries differing significantly in rates of D-SIB $\left[\chi^{2}(10)=144.45 ; p<.001\right]$. Prevalence for repetitive D-SIB ranged from $2.68 \%$ to $12.95 \%$ also showing significant differences between countries $\left[\chi^{2}(10)=191.92 ; p<.001\right]$.

The proportions of different forms of D-SIB for each gender are presented in Table 2. Skin damage, by using 'other' methods, was most commonly reported, followed by self-cutting. Self-burning and self-hitting were less common. The proportion of self-cutting and skin damage by using other methods 


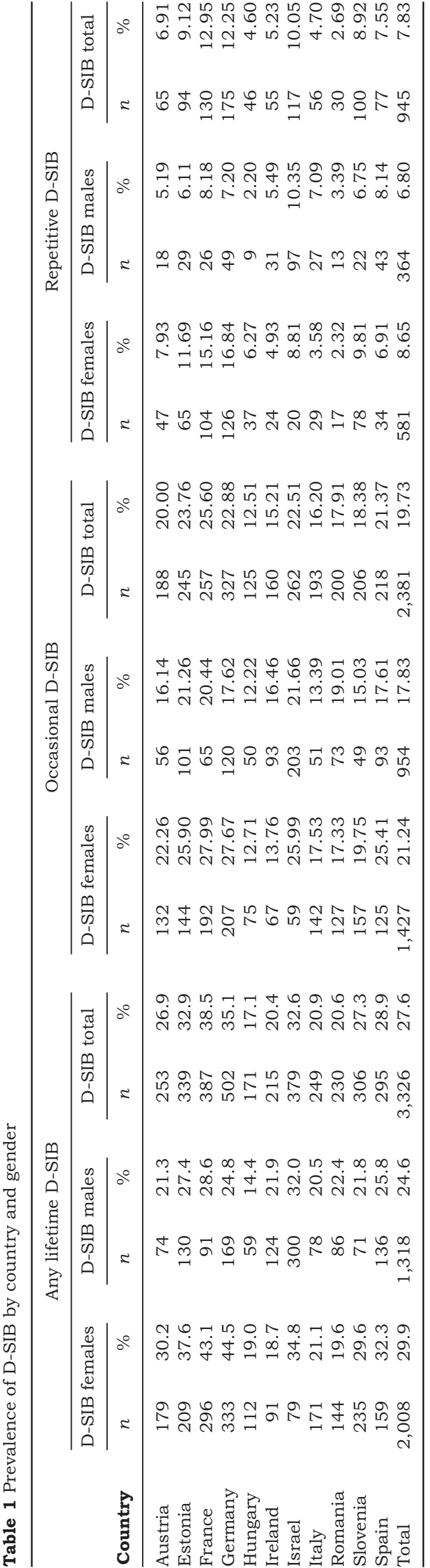

Table 2 Proportion of self-harm methods in adolescents with any lifetime D-SIB

\begin{tabular}{|c|c|c|c|c|c|}
\hline \multirow[b]{2}{*}{ Methods $^{a}$} & \multicolumn{3}{|c|}{$\begin{array}{l}\text { Frequency of methods } \\
(\%)\end{array}$} & \multicolumn{2}{|c|}{ Gender differences } \\
\hline & Females & Males & Total & OR & $95 \% \mathrm{CI}$ \\
\hline Self-cutting & 54.4 & 34.2 & 46.4 & $2.30 * *$ & $2.00-2.66$ \\
\hline Self-burning & 15.9 & 33.4 & 22.8 & $0.38 * *$ & $0.32-0.44$ \\
\hline Self-hitting & 25.4 & 34.3 & 28.9 & $0.65^{* *}$ & $0.56-0.76$ \\
\hline $\begin{array}{l}\text { Skin damage } \\
\text { by using other } \\
\text { methods }^{\mathrm{b}}\end{array}$ & 68.9 & 59.3 & 65.1 & $1.53^{* *}$ & $1.32-1.76$ \\
\hline
\end{tabular}

${ }^{\mathrm{a}}$ Multiple answers possible.

${ }^{\mathrm{b}}$ Self-scratching, -carving, -biting, or preventing wounds from healing.

${ }^{* *} p<.01$.

was higher among female students. Both self-burning and self-hitting, however, were less frequent among females.

\section{Psychosocial correlates of D-SIB}

Table 3 gives an overview of the distribution of demographic and psychological factors among the different D-SIB groups. Demographic and psychosocial correlates as predictors of D-SIB are presented in Table 4. Strongest predictors of D-SIB in the univariate regression (RRR ranging from 6.31 to 1.67), that also showed an independent effect in the multivariate regression model, are as follows (in hierarchical order of the strength of effect sizes): suicidality, anxiety and depressive symptoms, illegal drug consumption, peer victimization, sensation-seeking and delinquent behaviors, tobacco use, alcohol consumption, 'parents do not understand student's problems', 'parents to not pay attention to student', and parental unemployment. Male gender and being a religious person negatively predicted D-SIB. Age was positively predictive for D-SIB in the univariate regression, but negatively predictive for D-SIB in the multivariate model. The effects of household composition, immigration status and loneliness/peer relationship problems on D-SIB were sufficiently explained by the other variables in the multivariate regression models.

With regards to differentiation between occasional and repetitive D-SIB, both showed high levels of significance for all psychosocial variables in the univariate regression model. RRRs were higher for repetitive D-SIB (RRRs ranging from 15.45 to 1.12 ) compared with occasional D-SIB (RRRs ranging from 4.11 to 1.07$)$. In the multivariate regression model, occasional D-SIB was not associated with illegal drug consumptions and loneliness / peer relationship problems compared with repetitive D-SIB. In contrast with occasional D-SIB, repetitive D-SIB was not associated with gender. 
Table 3 Frequencies of demographic and psychological factors by D-SIB-group

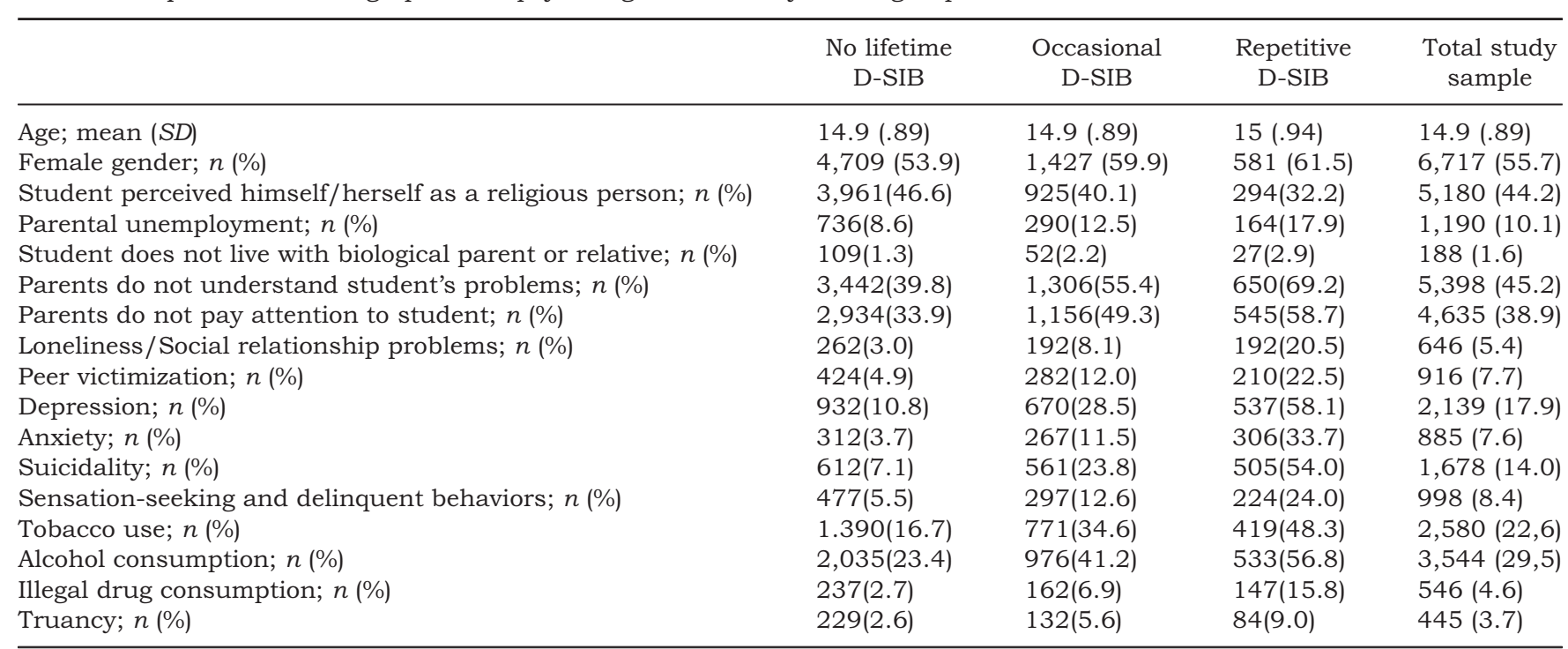

\section{Interactions of psychosocial correlates and gender}

Significant psychosocial variable $\times$ gender interactions were found for 'parents do not understand student's problems' $(p=.026)$, use of tobacco $(p=.026)$, alcohol consumption $(p=.019)$, and illegal drug consumption $(p=.015)$. For these psychosocial variables, female students were at significantly higher risk of engaging in D-SIB when reporting those psychosocial correlates compared with males.

\section{Interactions of psychosocial correlates and countries}

Significant psychosocial variable $\times$ country interactions were found for several psychosocial variables and are presented in Table 5. 'Student does not live with biological parent or relative', anxiety, tobacco use, illegal drug consumption, and truancy did not show significant country interactions.

\section{Medical treatment after D-SIB}

Table 6 presents the percentage of students who ever had to get medical treatment after an incident of D-SIB for each group. Interestingly, there was a gender $\left[\chi^{2}(1)=10.97 ; p<.001\right]$ and country $\left[\chi^{2}(10)=\right.$ 39.90; $p<.001]$ specific effect regarding the medical treatment. In the whole sample, males more frequently received medical treatment following D-SIB; however, this was not true in all countries. In Israel, medical treatment after incidents of D-SIB was most common and least common in France (Table 6). There was no gender $\times$ country interaction.

\section{Discussion}

This study assessed prevalence and psychosocial correlates of D-SIB, within the same timeframe and with a homogenous methodology, in a large multi- national sample of European adolescents. As recently published, the SEYLE sample can be considered reasonably representative for the adolescent population of their respective country (Carli et al., 2013).

\section{Prevalence}

Overall lifetime prevalence of D-SIB was $27.6 \%$, which lies within the range previously reported in epidemiological studies among adolescents (Nock, 2010). The prevalence of occasional D-SIB was $19.7 \%$ with significantly lower number of adolescents $(7.8 \%)$ reporting to engage in repetitive D-SIB. Significant differences in the frequency of D-SIB were found among the participating countries. These results confirm the large variation in prevalence estimates previously reported (Nock, 2010), and are consistent with previous studies reporting country differences in self-harming behavior among adolescent samples in Europe (Kokkevi et al., 2012; Madge et al., 2008; Portzky, De Wilde, \& Van Heeringen, 2008). The results differ, however, from population-based samples of adolescents -from Germany and from the United States - using the same sampling and assessment methods of NSSI (Plener et al., 2009). This study reported no significant differences regarding the frequency of NSSI, and their results were confirmed by another cross-national study of community samples from Italy, the Netherlands, and the United States (Giletta et al., 2012). It may be of note that all studies including a concept of self-harm regardless of suicidal intent have reported country differences (including our study), whereas studies focusing on NSSI did not.

A comparison of prevalence data from the Child \& Adolescent Self-harm in Europe (CASE) study with the present study indicates both similarities and differences. The main and most striking difference is that reported lifetime prevalence, in general, was 


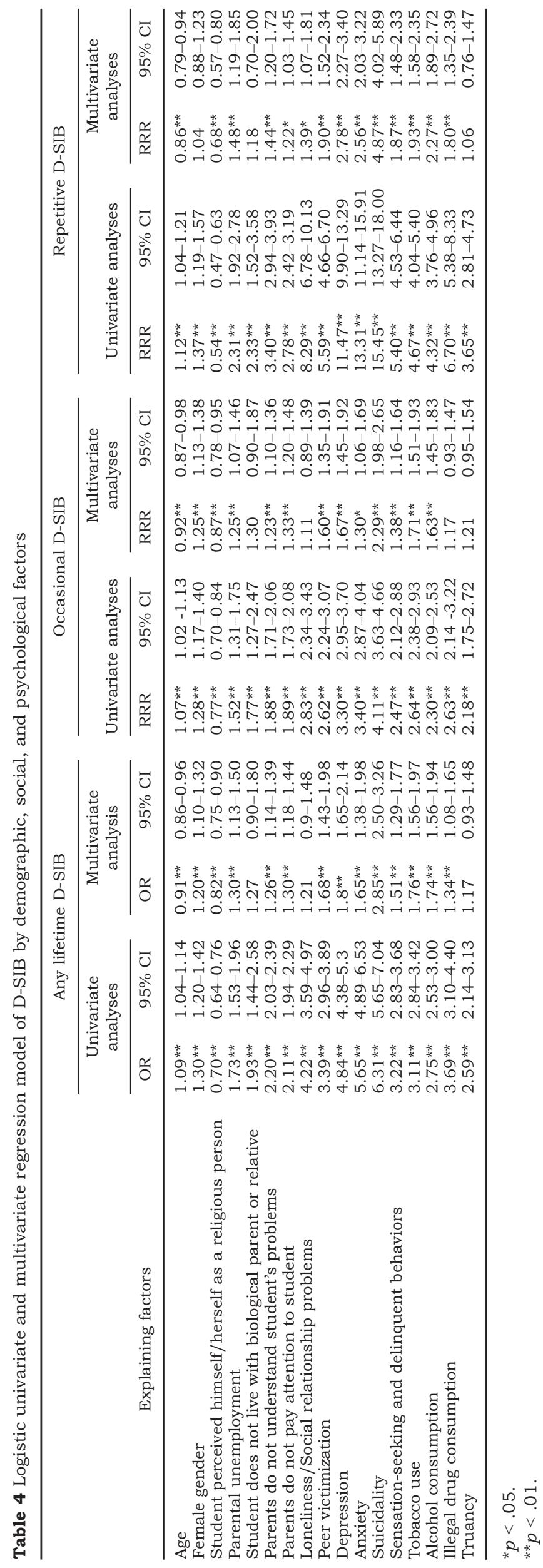

much lower in the CASE study performed in 2006, namely, $13.5 \%$ for girls and $4.3 \%$ for boys across their European sample (Madge et al., 2008). It is possible that prevalence rates reported in the present study of $29.9 \%$ for girls and $24.6 \%$ for boys reflect an increase from 2006 to 2010 in self-harming behavior across Europe, particularly among boys. However, the CASE and SEYLE samples overlapped only in two countries (Hungary and Ireland), the age range was different (in SEYLE students were 2 years younger) and different assessment tools were used (the CASE did investigate DSH incl. suicide attempts), which decreases comparability. Nevertheless, most comparisons (young versus old, D-SIB vs. DSH) would be expected to result in lower lifetime prevalence within the SEYLE sample, which is not the case.

\section{Gender differences}

Female adolescents reported higher frequencies of engaging in D-SIB compared with male adolescents. However, gender differences in D-SIB were not found in all study countries, suggesting cultural influence, as confirmed by significant country-gender interactions. Higher prevalence rates of self-injury in females seems to be a very consistent finding both in population-based samples (Brunner et al., 2007; O'Connor et al., 2009; Plener et al., 2009), as well as in clinical samples (Kirkcaldy, Brown, \& Siefen, 2006). The reasons for these gender differences are not yet clear. It has been argued that higher rates of depression and anxiety in girls could account for this (Hilt et al., 2008). However, we found that repetitive self-injury seems to be associated with severe emotional and behavioral problems in both genders. We also found that increased age on its own was a predictor of lifetime D-SIB (as expected); and younger age predicted D-SIB when controlling for all other psychosocial variables. A possible interpretation could be that younger adolescents with a similar load of psychosocial risk factors may be at higher risk of harming themselves.

The majority of adolescents from this study reported using multiple methods of self-injurious acts besides 'self-cutting', which is in line with former results (Whitlock et al., 2006). Gender differences were found for most of the methods reported. Girls reported higher frequency of overall D-SIB and self-cutting. In contrast, there was a gender-specific over-representation of self-burning and self-hitting in male adolescents.

\section{Psychopathology}

Consistent with previous studies, significant associations with depressive and anxiety symptoms were found in adolescents who engaged in D-SIB (Brunner et al., 2007; Hawton, Rodham, Evans, \& Weatherall, 2002; Lloyd-Richardson et al., 2007). These 
Table 5 Psychosocial variables showing significant interactions with country in the univariate regression model of D-SIB. In addition, both range and difference of change in probability of D-SIB [p(D-SIB)] by psychosocial variable are presented including the country with the lowest and highest change in $\mathrm{p}$ (D-SIB)

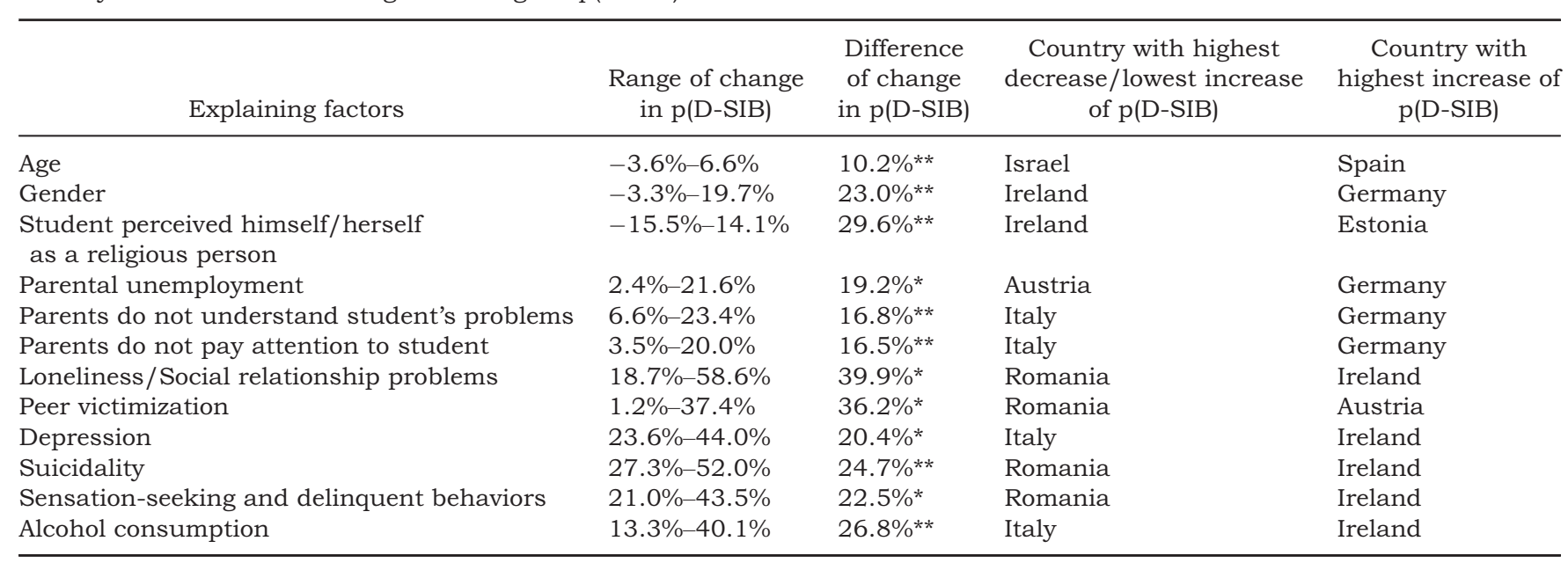

${ }^{*} p<.05$

${ }^{* *} p<.01$

Table 6 Medical treatment in the group of adolescents with D-SIB

\begin{tabular}{|c|c|c|c|c|c|}
\hline \multirow[b]{2}{*}{ Country } & \multicolumn{3}{|c|}{$\begin{array}{c}\text { Frequency of medical } \\
\text { treatment }(\%)\end{array}$} & \multicolumn{2}{|c|}{ Gender differences } \\
\hline & Females & Males & Total & OR & $95 \% \mathrm{CI}$ \\
\hline Austria & 1.35 & 0.58 & 1.06 & 2.36 & $0.50-11.19$ \\
\hline Estonia & 1.80 & 1.05 & 1.46 & 1.72 & $0.58-5.07$ \\
\hline France & 1.46 & 0.63 & 1.20 & 2.3 & $0.51-10.68$ \\
\hline Germany & 2.81 & 3.68 & 3.22 & 0.76 & $0.42-1.37$ \\
\hline Hungary & 1.02 & 0.74 & 0.90 & 1.39 & $0.34-5.58$ \\
\hline Ireland & 0.41 & 2.32 & 1.44 & $0.17^{*}$ & $0.04-0.78$ \\
\hline Israel & 3.98 & 6.10 & 5.69 & 0.64 & $0.31-1.31$ \\
\hline Italy & 0.37 & 1.57 & 0.76 & $0.23^{*}$ & $0.06-0.94$ \\
\hline Romania & 0.68 & 1.04 & 0.81 & 0.65 & $0.17-2.44$ \\
\hline Slovenia & 1.51 & 2.78 & 1.88 & 0.54 & $0.22-1.29$ \\
\hline Spain & 0.81 & 3.80 & 2.36 & $0.21^{* *}$ & $0.07-0.61$ \\
\hline Total & 1.34 & 2.74 & 1.96 & $0.48 * *$ & $0.37-0.63$ \\
\hline
\end{tabular}

${ }^{*} p<.05$.

$* * p<.01$

findings indicate that D-SIB is strongly indicative of psychological problems that require professional attention. A strong relationship between D-SIB and suicidality (i.e., suicidal thoughts and suicide attempts) was shown in this study, as well as in former studies (Brunner et al., 2007; Nock, Joiner, Gordon, Lloyd-Richardson, \& Prinstein, 2006). This relationship requires further investigation to address the current debate about whether self-injuries should be considered on a continuum of self-harm or regarded as a phenomenon separate from suicidal behavior (Nock, 2010). Joiner (2005) sees self-harm as a moderator that may increase the likelihood of suicidal thoughts being translated into actions. Consistent with this theory, longitudinal research showed that DSH is strongly associated with future suicide attempts (Cooper et al., 2005). In addition, the strong association with a broad range of risk-behaviors (substance use, sensation-seeking, and delinquent behaviors) has clinical implications, as D-SIB may be indicative of other risk-taking and self-destructive behaviors. These associations have been demonstrated in previous studies (Brunner et al., 2007), but whether risk-behavior may serve similar functions as D-SIB (e.g., to regulate intense negative emotional states) should be a focus of future research. The question whether D-SIB (e.g., self-cutting) and indirect harmful behaviors (e.g., substance abuse) should be considered on a continuum of self-harm has been raised by Holinger (1979) and Nock (2010), who proposed a model on a 'continuum of self-destructiveness' in adolescents.

\section{Psychosocial factors}

The strong association between peer victimization and self-reported D-SIB emphasizes the crucial role of interpersonal factors, in addition to intrapersonal factors, in the occurrence of D-SIB among adolescents (Klonsky, 2007). To avoid punishment or to get attention have been described as important interpersonal functions of self-injury (Nock \& Prinstein, 2004). Family related factors, like reduced parental involvement, but also parental unemployment, were strongly associated with D-SIB in our study, whereas family related factors like disruption of family composition did not show a strong relationship with D-SIB. These findings indicate that the quality of relationships within the family environment is a very important concomitant of self-harm, as stated in previous reports (Kaess et al., 2012; Wedig \& Nock, 2007; Yates, Tracy et al., 2008).

It needs to be noted that in this study, the associations between psychological variables and D-SIB were much stronger for repetitive D-SIB compared with occasional D-SIB. However, all psychosocial variables also showed significant 
associations with regards to occasional D-SIB. Given the large study sample, statistical significance should be interpreted cauciously. While variables with high RRR's like suicidality, anxiety, and depressive symptoms (all OR $>3$ ) seem to have large effects and may be of important clinical relevance in many cases, other variables (e.g. parental unemployment, parental attention to student) with lower RRR's may only have small effects, however, may be equally clinically important in the individual case.

In the present study, gender interactions have been found for variables related with parental involvement, as well as alcohol and substance abuse. The stronger association between lack of parental involvement and D-SIB in females may suggest a gender-specific vulnerability to lack of care or relationship difficulties with parents. A higher sensitivity to parent-child relationship has previously been reported for females with respect to suicidal behavior (Ponnet et al., 2005). Also, girls who engage in alcohol and substance abuse seem to be at higher risk of D-SIB compared with their male peers. Finally, male gender remained a significantly negative predictor of D-SIB when adjusting for all psychosocial variables in the multivariate model, which may suggest at least some gender-specific vulnerability for D-SIB.

\section{Intercultural variations}

Psychosocial correlates of self-harm thoughts have recently been reported to show intercultural variations. Kokkevi et al. (2012) found that substance abuse was more predictive for suicide attempts when it was reported in countries with a generally low prevalence of substance abuse. Our study is the first to show that several psychosocial risk factors of D-SIB have high intercultural variability. In Germany, for example, variables of family environment had a very strong influence on D-SIB, whereas risk behaviors and psychopathology showed the highest influence in Ireland. In Romania, only a marginal influence of psychopathology and risk behavior on D-SIB was present, whereas parenting variables showed almost no influence on D-SIB in Italy. The results on country interactions are very complex, and may be difficult to interpret. However, they indicate that cultural differences may strongly impact the relevance and influence of certain risk factors, which should be taken into account in prevention and clinical intervention among adolescents engaging in self-injury.

\section{Medical treatment}

Only a small minority of the affected adolescents reported to have had received medical treatment after D-SIB, which is in agreement with several other population-based studies (Deliberto \& Nock, 2008; Hawton et al., 2002; Ystgaard et al., 2009). The reason for this can be that the D-SIB physical injuries are often mild and kept in secret as self-harmers find consolation in peers with similar behaviors, which constitutes a challenge to the public health system to identify those who self-harm (Heath, Ross, Toste, Charlebois, \& Nedecheva, 2009; Sanci, Lewis, \& Patton, 2010; Tylee, Haller, Graham, Churchill, \& Sanci, 2007). The observed gender differences in our study may show that males are likely to engage in more severe D-SIB, which could consequently lead to higher rates of medical treatment. A gender pattern of lower frequency but higher severity among males is a well-known phenomenon, based on suicide attempts among adolescents (Beautrais, 2002).

In general, our findings suggest that both repetitive and occasional D-SIB require professional attention, but this is not to say that all young people with occasional self-injury need mental health treatment. A study from the general population (Moran et al., 2012) indicates that the majority of young people's self-injury behavior will remit in short periods of time, but other findings (e.g. Bergen et al., 2012; Wilkinson, Kelvin, Roberts, Dubicka, \& Goodyer, 2011) suggest that a history of self-harm is an important clinical marker for subsequent suicide attempts, other negative health outcomes and death. Therefore, young people with occasional D-SIB might at least deserve further investigation and examination of their mental health status. Future longitudinal studies are needed to identify young people with risk constellation who require mental health care or other preventive interventions according to a staging model (McGorry, Hickie, Yung, Pantelis, \& Jackson, 2006). Further research on specialized treatments for self-harming adolescents is critical (Fischer, Parzer, Resch, Brunner, \& Kaess, 2013) as there are currently no independently replicated efficacious interventions available (Ougrin, Tranah, Leigh, Taylor, \& Asamow, 2012).

\section{Limitations and strengths}

A limitation of these analyses is that the prevalence estimates of D-SIB, as well as the assessment of all psychosocial variables in this study, relied on self-report. However, standardized and validated instruments were used in SEYLE, although our modified 6-item D-SIB questionnaire did not distinguish between direct self-injury with and without suicidal intent, which may be another limitation. It needs to be noted that judgment of suicidal intent of self-harming acts by adolescents' self-report is questionable and may lack reliability. The fact that the study reports on a cross-sectional analyses is another limitation. Only longitudinal data can give information about causality.

A major strength of this study is the large nonclinical population-based sample of adolescents, recruited from randomly selected schools, across 
eleven study sites, which were reasonably representative of their respective European country (Carli et al., 2013). The students were recruited and evaluated in each country with homogenous procedures and measurements. Furthermore, to our knowledge, this study of adolescent lifetime D-SIB comprises the largest geographic area distributed over many countries ever reported.

\section{Conclusion}

More than every fourth adolescent in Europe was found to have engaged in D-SIB during lifetime; females showing higher rates of occasional selfinjury, with a special preference for self-cutting.

Suicidality demonstrated the strongest association with D-SIB, which confirms the role of direct self-injury as a possible indicator or mediator of adolescent suicidal behavior. A strong association of D-SIB was also shown with other psychopathology, risk behaviors, family related problems and neglect, as well as peer-related rejection/victimization.

The majority of adolescents who engaged in D-SIB did not receive any professional help nor did they seek help after their self-injury. Although this may often be due to mild injuries, it is important to enhance our understanding of the barriers in receiving specialized treatment services. In addition, there is an urgent need to improve treatment development and research in the field of adolescent self-harm.

Across the eleven European countries, the frequency of D-SIB was wide ranged, which calls not only for psychiatric and medical, but also for multidimensional explanations including social and anthropological studies on cultural differences.

\section{Supporting information}

Additional Supporting Information may be found in the online version of this article:

Table S1. Cut-offs in the SEYLE study for adolescent risk-behaviour and psychopathology

Table S2. Odds ratios of the prevalence of total D-SIB by country

\section{Acknowledgments}

The SEYLE project is supported by the European Union through the Seventh Framework Program (FP7), Grant agreement number HEALTH-F2-2009-223091. SEYLE Project Leader and Principal Investigator is Professor in Psychiatry and Suicidology Danuta Wasserman (D.W.), National Centre for Suicide Research and Prevention of Mental Ill-Health (NASP) Karolinska Institutet, Stockholm, Sweden. The Executive Committee comprises Danuta Wasserman and Vladimir Carli, both from NASP, Sweden; Marco Sarchiapone, University of Molise, Campobasso, Italy; Christina W. Hoven, and Camilla Wasserman, both from Columbia University, NY, USA; the SEYLE Consortium comprises sites in 12 European countries. Site leaders are Danuta Wasser- man (NASP, Coordinating Centre), Christian Haring (Austria), Airi Varnik (Estonia), J-.P. Kahn (France), Romuald Brunner (Germany), Judit Balazs (Hungary), Paul Corcoran (Ireland), Alan Apter (Israel), Marco Sarchiapone (Italy), Donia Cosman (Romania), Dragan Marusic/Vita Postuvan (Slovenia), and Julio Bobes (Spain).

In addition, special thanks to: Katja Klug, Judith Frisch, Lisa Göbelbecker, and Sarah Schneider from the University of Heidelberg in Germany; Mária Bálint, Ágnes Keresztény, Luca Farkas, and Julia Gádoros from Hungary; the Estonian Ministry of Social Affairs for the financial support and all researchers and other staff participating in the implementation of the SEYLE project in Estonia, in particular Peeter Värnik and Mari Jushkin; at the University of Oviedo-CIBERSAM, Ma Paz García-Portilla, Manuel Bousoño, Susana Al-Halabí, Ma Teresa Bascarán, Eva Ma Díaz-Mesa, Marlen Garrido, Patricia Buron, Jose Luis Rancaño, and Gonzalo Galvan; Miriam Iosue, Marianna D’Aulerio, and Francesco Basilico at University of Molise in Italy.

The authors were independent of the funders in all aspects of study design, data analysis, and writing of the manuscript.

\section{Correspondence}

Michael Kaess, U.D. Section for Disorders of Personality Development, Department of Child and Adolescent Psychiatry, Center for Psychosocial Medicine, University of Heidelberg, Blumenstrasse 8, 69115 Heidelberg, Germany; Email: michael.kaess@med. uni-heidelberg.de

\section{References}

American Psychiatric Association (2013). Diagnostic and statistical manual of mental disorders: Fifth edition (DSM-5). Arlington, VA: American Psychiatric Publishing.

Beautrais, A.L. (2002). Gender issues in youth suicidal behavior. Emergency Medicine, 14, 35-42.

Beck, A.T., Steer, R.A., Ball, R., \& Ranieri, W. (1996). Comparison of Beck Depression Inventories -IA and -II in psychiatric outpatients. Journal of Personality Assessment, 67, 588-597.

Bergen, H., Hawton, K., Waters, K., Ness, J., Cooper, J., Steeg, S., \& Kapur, N. (2012). Premature death after self-harm: A multicentre cohort study. The Lancet, 380, 1568-1574.

Bjärehed, J., \& Lundh, L.G. (2008). Deliberate self-harm in 14-year-old adolescents: How frequent is it, and how is it associated with psychopathology, relationship variables, and styles of emotional regulation? Cognitive Behaviour Therapy, 37, 26-37.

Brunner, R., Parzer, P., Haffner, J., Steen, R., Roos, J., Klett, M., \& Resch, F. (2007). Prevalence and psychological correlates of occasional and repetitive deliberate self-harm in adolescents. Archives of Pediatrics and Adolescent Medicine, 161, 641-649.

van Buuren, S., \& Oudshoorn, C.G.M. (1999). Flexible multivariate imputation by MICE. TNO-rapport PG 99.054. Leiden: TNO Prevention and Health.

Carli, V., Wasserman, C., Wasserman, D., Sarchiapone, M., Apter, A., Balazs, J., ... \& Hoven, C.W. (2013). The Saving and Empowering Young Lives in Europe (SEYLE) Randomized Controlled Trial (RCT): Methodological issues and participant characteristics. BMC Public Health, 13, 479.

Cooper, J., Kapur, N., Webb, R., Lawlor, M., Guthrie, E., Mackway-Jones, K., \& Appleby, L. (2005). Suicide after 
deliberate self-harm: A 4-year cohort study. American Journal of Psychiatry, 162, 297-303.

Deliberto, T.L., \& Nock, M.K. (2008). An exploratory study of correlates, onset, and offset of non-suicidal self-injury. Archives of Suicide Research, 12, 219-231.

EVS (2009). The European values study questionnaire. The Netherlands: Tillburg University.

Fischer, G., Parzer, P., Resch, F., Brunner, R., \& Kaess, M. (2013). Short-term psychotherapeutic treatment in adolescents with non-suicidal self-injury - a randomized controlled trial. Trials, 14, 294.

Giletta, M., Scholte, R.H., Engels, R.C., Ciairano, S., \& Prinstein, M.J. (2012). Adolescent non-suicidal self-injury: A cross-national study of community samples from Italy, the Netherlands and the United States. Psychiatry Research, 197, 66-72.

Gollust, S.E., Eisenberg, D., \& Golberstein, E. (2008). Prevalence and correlates of self-injury among university students. Journal of American College Health, 56, 491-498.

Gratz, K. (2001). Measurement of deliberate self-harm: Preliminary data on the Deliberate Self-Harm Inventory. Journal of Psychopathology and Behavioral Assessment, 23, 253-263.

Haw, C., Hawton, K., Casey, D., Bale, E., \& Shepherd, A. (2005). Alcohol dependence, excessive drinking and deliberate self-harm: Trends and patterns in Oxford, 1989-2002. Social Psychiatry and Psychiatric Epidemiology, 40, 964-971.

Hawton, K., \& James, A. (2005). Suicide and deliberate self harm in young people. BMJ, 330, 891-894.

Hawton, K., Rodham, K., Evans, E., \& Weatherall, R. (2002). Deliberate self harm in adolescents: Self report survey in schools in England. BMJ, 325, 1207-1211.

Heath, N.L., Ross, S., Toste, J.R., Charlebois, A., \& Nedecheva, T. (2009). Retrospective analysis of social factors and nonsuicidal self-injury among young adults. Canadian Journal of Behavioural Science, 41, 180-186.

Hilt, L.M., Cha, C.B., \& Nolen-Hoeksema, S. (2008). Nonsuicidal self-injury in young adolescent girls: Moderators of the distress-function relationship. Journal of Consulting and Clinical Psychology, 76, 63-71.

Holinger, P.C. (1979). Violent deaths among the young: Recent trends in suicide, homicide, and accidents. American Journal of Psychiatry, 136, 1144-1147.

Joiner, T.E. (2005). Why people die by suicide. Cambridge MA: Harvard University Press.

Kaess, M., Parzer, P., Mattern, M., Plener, P.L., Bifulco, A., Resch, F., \& Brunner, R. (2013). Adverse childhood experiences and their impact on frequency, severity, and the individual function of nonsuicidal self-injury in youth. Psychiatry Research, 206, 265-272.

Kaess, M., Parzer, P., Mattern, M., Resch, F., Bifulco, A., \& Brunner, R. (2012). [Childhood Experiences of Care and Abuse (CECA) - validation of the German version of the questionnaire and interview, and results of an investigation of correlations between adverse childhood experiences and suicidal behavior]. Z Kinder Jugendpsychiatr Psychother, 39, 243-252.

Kapur, N., Cooper, J., O'connor, R.C., \& Hawton, K. (2013). Non-suicidal self-injury v. attempted suicide: New diagnosis or false dichotomy? British Journal of Psychiatry, 202, 326-328.

Kirkcaldy, B.D., Brown, J., \& Siefen, R.G. (2006). Disruptive behavioral disorders, self harm and suicidal ideation among German adolescents in psychiatric care. International Journal of Adolescent Medicine and Health, 18, 597-614.

Klonsky, E.D. (2007). The functions of deliberate self-injury: A review of the evidence. Clinical Psychology Review, 27, 226-239.

Kokkevi, A., Rotsika, V., Arapaki, A., \& Richardson, C. (2012). Adolescents' self-reported suicide attempts, self-harm thoughts and their correlates across 17 European countries. Journal of Child Psychology and Psychiatry, 53, 381-389.

Lloyd-Richardson, E.E., Perrine, N., Dierker, L., \& Kelley, M.L. (2007). Characteristics and functions of non-suicidal self-injury in a community sample of adolescents. Psychological Medicine, 37, 1183-1192.

Lundh, L.G., Karim, J., \& Quilisch, E. (2007). Deliberate self-harm in 15-year-old adolescents: A pilot study with a modified version of the Deliberate Self-Harm Inventory. Scandinavian Journal of Psychology, 48, 33-41.

Madge, N., Hewitt, A., Hawton, K., De Wilde, E.J., Corcoran, P., Fekete, S., ... \& Ystgaard, M. (2008). Deliberate self-harm within an international community sample of young people: Comparative findings from the Child \& Adolescent Self-harm in Europe (CASE) Study. Journal of Child Psychology and Psychiatry, 49, 667-677.

McGorry, P.D., Hickie, I.B., Yung, A.R., Pantelis, C., \& Jackson, H.J. (2006). Clinical staging of psychiatric disorders: A heuristic framework for choosing earlier, safer and more effective interventions. Australian and New Zealand Journal of Psychiatry, 40, 616-622.

Moran, P., Coffey, C., Romaniuk, H., Olsson, C., Borschmann, R., Carlin, J.B., \& Patton, G.C. (2012). The natural history of self-harm from adolescence to young adulthood: A population-based cohort study. The Lancet, 379, 236-243.

Muehlenkamp, J.J., Claes, L., Havertape, L., \& Plener, P.L. (2012). International prevalence of adolescent non-suicidal self-injury and deliberate self-harm. Child and Adolescent Psychiatry and Mental Health, 6, 10.

Muehlenkamp, J.J., Kerr, P.L., Bradley, A.R., \& Adams Larsen, M. (2010). Abuse subtypes and nonsuicidal self-injury: Preliminary evidence of complex emotion regulation patterns. The Journal of Nervous and Mental Disease, 198, 258-263.

Nada-Raja, S., Skegg, K., Langley, J., Morrison, D., \& Sowerby, P. (2004). Self-harmful behaviors in a population-based sample of young adults. Suicide and Lifethreatening Behavior, 34, 177-186.

Nock, M.K. (2010). Self-injury. Annual Review of Clinical Psychology, 6, 339-363.

Nock, M.K., Joiner, T.E., Jr, Gordon, K.H., Lloyd-Richardson, E., \& Prinstein, M.J. (2006). Non-suicidal self-injury among adolescents: Diagnostic correlates and relation to suicide attempts. Psychiatry Research, 144, 65-72.

Nock, M.K., \& Prinstein, M.J. (2004). A functional approach to the assessment of self-mutilative behavior. Journal of Consulting and Clinical Psychology, 72, 885-890.

O'Connor, R.C., Rasmussen, S., Miles, J., \& Hawton, K. (2009). Self-harm in adolescents: Self-report survey in schools in Scotland. British Journal of Psychiatry, 194, 68-72.

Ougrin, D., Tranah, T., Leigh, E., Taylor, L., \& Asamow, J.R. (2012). Practitioner review: Self-harm in adolescents. Journal of Child Psychology and Psychiatry, 53, 337-350.

Paykel, E.S., Myers, J.K., Lindenthal, J.J., \& Tanner, J. (1974). Suicidal feelings in the general population: A prevalence study. British Journal of Psychiatry, 124, 380-406.

Plener, P.L., Libal, G., Keller, F., Fegert, J.M., \& Muehlenkamp, J.J. (2009). An international comparison of adolescent non-suicidal self-injury (NSSI) and suicide attempts: Germany and the USA. Psychological Medicine, 39, 1549-1558.

Ponnet, K., Vermeiren, R., Jespers, I., Mussche, B., Ruchkin, V., Schwab-Stone, M., \& Deboutte, D. (2005). Suicidal behavior in adolescents: Associations with parental marital status and perceived parent-adolescent relationship. Journal of Affective Disorders, 89, 107-113.

Portzky, G., De Wilde, E.J., \& Van Heeringen, K. (2008). Deliberate self-harm in young people: Differences in prevalence and risk factors between the Netherlands and Belgium. European Child and Adolescent Psychiatry, 17, 179-186. 
Rubin, D.B. (2004). Multiple imputation for nonresponse in surveys. New York: Wiley.

Sanci, L., Lewis, D., \& Patton, G. (2010). Detecting emotional disorder in young people in primary care. Current Opinion Psychiatry, 23, 318-323.

Serras, A., Saules, K.K., Cranford, J.A., \& Eisenberg, D. (2010). Self-injury, substance use, and associated risk factors in a multi-campus probability sample of college students. Psychology of Addictive Behaviors, 24, 119-128.

Tylee, A., Haller, D.M., Graham, T., Churchill, R., \& Sanci, L.A. (2007). Youth-friendly primary-care services: How are we doing and what more needs to be done? The Lancet, 369, $1565-1573$

Wasserman, D., Carli, V., Wasserman, C., Apter, A., Balazs, J., Bobes, J., ... \& Hoven, C.W. (2010). Saving and empowering young lives in Europe (SEYLE): A randomized controlled trial. BMC Public Health, 10, 192.

Wedig, M.M., \& Nock, M.K. (2007). Parental expressed emotion and adolescent self-injury. Journal of the American Academy of Child and Adolescent Psychiatry, 46, 1171-1178.

Weierich, M.R., \& Nock, M.K. (2008). Posttraumatic stress symptoms mediate the relation between childhood sexual abuse and nonsuicidal self-injury. Journal of Consulting and Clinical Psychology, 76, 39-44.

Whitlock, J., Eckenrode, J., \& Silverman, D. (2006). Self-injurious behaviors in a college population. Pediatrics, 117, 1939-1948.
WHO (2009). Global school-based student health survey. Geneva: World Health Organisation.

Wilkinson, P., Kelvin, R., Roberts, C., Dubicka, B., \& Goodyer, I. (2011). Clinical and psychosocial predictors of suicide attempts and nonsuicidal self-injury in the Adolescent Depression Antidepressants and Psychotherapy Trial (ADAPT). American Journal of Psychiatry, 168, 495501

Yates, T.M., Carlson, E.A., \& Egeland, B. (2008). A prospective study of child maltreatment and self-injurious behavior in a community sample. Development and Psychopathology, 20, 651-671.

Yates, T.M., Tracy, A.J., \& Luthar, S.S. (2008). Nonsuicidal self-injury among "privileged" youths: Longitudinal and cross-sectional approaches to developmental process. Journal of Consulting and Clinical Psychology, 76, 52-62.

Ystgaard, M., Arensman, E., Hawton, K., Madge, N., Van Heeringen, K., Hewitt, A., ... \& Fekete, S. (2009). Deliberate self-harm in adolescents: Comparison between those who receive help following self-harm and those who do not. Journal of Adolescence, 32, 875-891.

Zung, W.W. (1971). A rating instrument for anxiety disorders. Psychosomatics, 12, 371-379.

Accepted for publication: 28 August 2013

Published online: 12 November 2013 